\title{
Experiencia del internado de medicina durante la pandemia por Covid-19
}

\author{
Medical internship experience during the Covid-19 pandemic
}

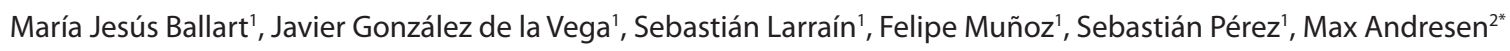

\begin{abstract}
Resumen
Introducción: la pandemia por SARS-CoV-2 ha causado cambios en la formación médica a nivel mundial. Se han suspendido todas las actividades presenciales buscando reducir el contagio y, además, dada la creciente demanda hospitalaria, ha generado la necesidad de buscar nuevas formas de mantener la docencia y a la vez, balancear ésta con las necesidades hospitalarias. Desarrollo: revisamos la experiencia de estudiantes de medicina en otros países, tanto las similitudes como diferencias respecto a lo vivido en Chile, bajo nuestra formación. El interno de medicina, a diferencia del estudiante o del médico, se encuentra en un rol intermedio como práctica profesional y consideramos que tiene un rol positivo en el apoyo a los servicios de salud durante la crisis actual. Presentamos nuestra experiencia como internos de medicina voluntarios en los distintos campos clínicos que se encuentran bajo el alero de nuestra universidad y como hemos experimentado la creciente sobrecarga hospitalaria, incertidumbre, soledad y experiencia con la muerte en nuestra práctica. Conclusión: la experiencia como internos durante esta pandemia y como voluntarios en los distintos servicios de nuestros campos clínicos ha alterado profundamente nuestra formación médica. Nos ha permitido interiorizarnos no solo en aspectos técnicos, sino también en el área más humana de la medicina, en aprender sobre trabajo en equipo, la incertidumbre y el fin de la vida. Creemos que todo esto nos ha preparado y nos ha dado una experiencia invaluable para nuestra futura práctica médica.
\end{abstract}

Palabras clave: COVID-19; SARS-COV-2; educación médica; estudiantes de medicina; Chile

\begin{abstract}
Introduction: The SARS-Cov-2 pandemic has changed medical training worldwide. All face-to-face activities have been suspended seeking to reduce contagion, and also, given the growing hospital demand, it has generated the need to find new ways to keep teaching and, at the same time, balance it with hospital needs. Body: We review the experience of medical students in other countries, both similarities and differences from what has been experienced in Chile under our training. Medical interns, unlike other students or physicians, are in an intermediate role in their professional practice, and we consider that we have a positive role in supporting health services during the current crisis. We present our experience as volunteer medical interns in the different clinical facilities that are associated with our university and how we have experienced the increasing hospital overload, uncertainty, loneliness, and experience with death in our practice. Conclusion: The experience as interns during this pandemic and as volunteers in the different departments from our clinical facilities has profoundly affected our medical training. It has allowed us to understand not only technical aspects but also the most humanitarian areas of medicine, by learning about teamwork, living with uncertainty, and the end of life. We believe that all of this has prepared us and given us invaluable experience for our future medical practice.
\end{abstract}

Keywords: COVID-19; SARS-COV-2; medical education; medical students; Chile

Fecha de Envío: 28 de julio de 2020 - Fecha de aceptación: 11 de septiembre de 2020

\section{Introducción}

La pandemia por severe acute respiratory syndrome coronavirus type 2 (SARS-CoV-2) ha cambiado el paradigma sobre la formación médica a nivel mundial. Ha llevado a replantear las mallas curriculares, balanceando la necesidad de egresar nuevos médicos versus que éstos logren los conocimientos mínimos para poder ejercer.

(1) Facultad de Medicina, Pontificia Universidad Católica de Chile, Santiago, Chile

(2) Departamento de Medicina Intensiva, Facultad de Medicina, Pontificia Universidad Católica de Chile.

*Correspondencia: andresen@med.puc.cl 
Algunos de los conflictos que se han presentado han sido la suspensión de clases presenciales, la pérdida de horas de rotaciones clínicas, especialmente en las especialidades que requieren el desarrollo de habilidades técnicas, la disminución de la exposición a pacientes con el consiguiente impacto en la relación médico paciente, la cancelación de congresos científicos e investigación como de la posposición y cambios en la forma de evaluaciones de estudiantes de medicina. Al mismo tiempo, los estudiantes enfrentan una situación complicada ante el deber quedarse en sus hogares para disminuir el contagio, vivir las consecuencias de la interrupción de sus estudios, exponiéndose a problemas de salud mental y por otra parte sentir un fuerte deseo, propio de la vocación, de querer ser partícipes y colaboradores en momentos de crisis sanitaria mundial. Esto ha generado una necesidad de crear nuevas formas de docencia en el área médica y desafíos para implementar opciones de corto plazo que les permitan a los médicos en formación el poder aprender y ayudar en estas circunstancias.

En otros países, como en Europa o América del Norte, las rotaciones clínicas fueron pausadas y los internos de último año, fueron egresados precozmente para responder a la creciente demanda de personal de salud (Chandratre, 2020; Kinder \& Harvey, 2020). Para estudiantes de otros cursos, la educación se reorganizó a través de formatos online y se ofrecieron voluntariados en centros de llamada para orientación a la comunidad sobre COVID-19 (Flotte et al., 2020; Gill et al., 2020; Kinder \& Harvey, 2020; Moszkowicz, et al., 2020; Rose, 2020; Roskvist, et al., 2020; Santos et al., 2020). En nuestro país, si bien no se realizó un egreso temprano, el Ministerio de Salud de Chile (MINSAL) decretó estado de emergencia, lo que permite a internos trabajar en forma remunerada para los Servicios de Salud con mayor necesidad de recurso humano. Por otro lado, las universidades chilenas formadoras suspendieron sus actividades académicas. En particular, la Pontificia Universidad Católica de Chile (PUC) tomó esta decisión el 16 de marzo. Al día siguiente, se realizó un llamado a sus estudiantes de último año a participar en forma voluntaria cumpliendo un rol asistencial en los diversos centros de salud asociados a ellas, de forma de responder a la pandemia. Esta llamada tuvo una rápida y positiva respuesta, completando los cupos en un par horas para cumplir con la demanda asistencial de los distintos servicios y centros.

En este artículo, queremos compartir la forma en que se nos permitió trabajar como internos voluntarios durante el período de mayor crisis sanitaria en relación al COVID-19, y las enseñanzas que esta experiencia nos deja.

\section{Internado en tiempos de COVID-19}

Al revisar las publicaciones anglosajonas sobre la experiencia y enfrentamiento de los estudiantes de medicina frente a la enfermedad por SARS-Cov-2 (COVID-19), se pueden evidenciar ciertas similitudes y diferencias respecto a lo vivido en Chile.

Como se mencionó previamente, la titulación precoz de muchos estudiantes fue un recurso utilizado por el hemisferio norte (Chandratre, 2020; Kinder \& Harvey, 2020), que al menos en nuestro país no se ha considerado aún. Esta decisión es claramente afectada por el hecho de que, en dichos países, los alumnos se encontraban en la segunda mitad del año, cercano al término definitivo de su carrera, a diferencia de nuestro caso, que recién comenzábamos a vivir nuestro último año de formación.

Por otra parte, encontramos que, en otros países, se incentivó la participación de estudiantes de medicina para apoyar de forma no presencial a los sistemas de salud, como por ejemplo la invitación a estudiantes de la Oschner Clinical School de Nueva Orleans a participar en la línea telefónica de su hospital, realizando triage y seguimiento de pacientes, e incluso colaborando en la recolección de datos para el estudio de prevalencia de COVID-19 de la propia institución (Santos et al., 2020). Una situación similar se vivió en nuestra Universidad, en donde se nos invitó, como internos, a formar parte de "Proyecto Esperanza" en su primera etapa (Call Center de la PUC), en donde cumpliríamos el rol de determinar qué pacientes que consultan telefónicamente tendrían indicación de asistir a un centro de salud a realizar un test de reacción en cadena de polimerasa (PCR) COVID-19. De esta forma, se intentaría descongestionar los servicios de urgencia, al lograr identificar aquellos pacientes con síntomas de poca severidad o dudas que pudiesen ser respondidas mediante un llamado telefónico.

Consideramos importante también, explicar lo que ha sido nuestra experiencia como voluntarios clínicos en este contexto de pandemia.

Existe escasa experiencia publicada sobre situaciones similares en la literatura disponible a la fecha, en donde más bien se describe la titulación temprana, o labores de apoyo no clínicas, además del cambio del aprendizaje hacia la modalidad online, a través de videoconferencias con docentes y resolución de casos clínicos online (Flotte et al, 2020; Gill et al., 2020; Kinder \& Harvey, 2020; Moszkowicz et al., 2020; Rose, 2020; Roskvist et al., 2020; Santos et al., 2020). Teniendo esto en consideración, nos parece pertinente describir brevemente el modelo que se utilizó y el cómo este se adecuó a las necesidades asistenciales de los centros formadores como a la educación continua de nosotros como alumnos de último año. 
En primer lugar, pese a la alta demanda de elementos de protección personal (EPP), y su eventual escasez, tal y como ha sido descrito en publicaciones en otros países (Rose, 2020), fue posible contar con EPP en nuestra práctica clínica. La PUC cuenta con distintos campos clínicos en las comunas de Santiago Centro, La Florida, Puente Alto, La Pintana y Las Condes en la Región Metropolitana, entre ellos centros de atención primaria y centros hospitalarios públicos y privados. En dichos centros, la dirección de pregrado de la escuela de medicina definió, a través de la consulta directa a los servicios de cada campo clínico, en cuáles de éstos se necesitaban internos como apoyo asistencial y el número necesario para cubrir las actividades clínicas. Luego, se presentó una oferta total de cupos a los estudiantes y cada uno, dentro de sus posibilidades y preferencias, podría asistir a cada servicio, en forma voluntaria, realizando períodos intermitentes de 2 semanas de ir al hospital, y 2 semanas en casa (modalidad on-off). Así funcionó entre finales de marzo hasta principios de junio. Las principales especialidades en donde desempeñamos nuestra labor fueron urgencias, medicina Interna y cuidados intensivos, acorde a la demanda durante la pandemia.

\section{Aprendizaje}

El rol del estudiante de medicina ha variado a lo largo de las instituciones y países. Consideramos importante recordar que el estudiante de medicina no es un trabajador remunerado, y más aún, no es médico. Sin embargo, los internos también se preocupan del cuidado clínico de los pacientes, realizando ciertas actividades propias de la labor médica, como entrevistar a los pacientes, responder llamados, dejar indicaciones médicas (siempre bajo supervisión), comunicarse con familiares, escribir evoluciones clínicas, realizar educación al paciente y su familia, asistir en procedimientos, y colaborar en los planes y decisiones clínicas que se toman sobre los pacientes.

Por un lado, desde el inicio de la pandemia ha sido un tema controversial la participación de alumnos de último año de medicina en la actividad asistencial, dado que pueden ser potenciales vectores de transmisión, al trabajar requieren uso de EPP y generan una carga extra para los clínicos docentes. Esto causó que ciertas escuelas de medicina tomaran la decisión de prohibir cualquier interacción de estos con pacientes. Sin embargo, en otras escuelas, incluyendo la PUC, se consideró que permitir que los estudiantes de medicina desempeñaran funciones clínicas era beneficioso para los pacientes, lo cual se alinea con el rol del estudiante de medicina sugerido en una publicación por Miller et al. (2020). Esto, debido a que los internos son versátiles en muchos roles clínicos, por lo que permitirles integrar equipos de salud puede mejorar la atención al paciente, contribuir a evitar crisis de personal, aumentar la eficiencia de un servicio hospitalario e incluso trabajar a distancia a través de telemedicina.

Durante la pandemia experimentamos diversas situaciones en diferentes servicios. En primer lugar, es importante recalcar que todos nosotros tuvimos las condiciones sociales y de salud adecuadas para participar como voluntarios en actividades clínicas que involucran contacto con pacientes, tanto confirmados como sospechosos.

La sobrecarga de pacientes en el sistema hospitalario dejó en evidencia que el trabajo en equipo es un aspecto central de la salud. A todos nos impresionó ver cómo administrativos, personal de aseo y de transporte, químicos farmacéuticos, tecnólogos médicos, técnicos en enfermería de nivel superior, enfermeras, kinesiólogos, nutricionistas, fonoaudiólogos y médicos de todas las especialidades y edades, trabajaron juntos para poder responder a la gran demanda de pacientes. Fuimos testigos de personal de salud que tuvo que reubicarse en nuevos servicios y adaptarse a éstos, un claro ejemplo fueron enfermeras pediátricas y médicos dermatólogos trabajando en unidades de cuidados intensivos de adultos. Esto demuestra la gran capacidad de adaptación y compromiso que tiene el personal de salud, y a través de los cuales, el aprendizaje fue enorme.

La pandemia por COVID-19 ha puesto en evidencia que el conocimiento en medicina es extremadamente dinámico. Numerosos paradigmas, especialmente de la ventilación mecánica, hoy han quedado atrás y han tenido que ser reformulados. Tuvimos que aprender a trabajar con gran incertidumbre, sin saber si los exámenes de laboratorio habitualmente utilizados predecían la evolución de los pacientes o si las terapias eran realmente efectivas.

Por otra parte, hemos vivido la experiencia de enfrentar la muerte en forma muy cercana, a un ritmo sin precedentes, la cual se ha visto severamente afectada por la soledad de nuestros pacientes. Lamentablemente, aunque de forma necesaria, se restringieron las visitas a los pacientes para evitar aumentar los contagios. Esta situación hace que encontremos a nuestros pacientes solos, en un momento tan vulnerable como lo es una hospitalización, más aún en una pandemia desconocida que los angustia y les genera incertidumbre, teniendo que vivir experiencias difíciles como una próxima intubación o en el período de fin de vida, sin la compañía de sus seres queridos.

\section{Conclusión}

Creemos que la pandemia por COVID-19 ha marcado profundamente nuestra formación médica, lo que se verá reflejado en nuestra práctica como médicos. Lo vivido nos ha permitido continuar en 
cierta medida con nuestro aprendizaje curricular, pero por sobre todo nos ha llevado a acercarnos y a aprender sobre temas más humanos, como trabajo en equipo, incertidumbre y fin de vida, los cuales en muchas ocasiones son dejados de lado dada la necesidad de cubrir un amplio abanico de conocimientos técnicos. Consideramos que durante todo este tiempo hemos logrado un nivel de conocimientos adecuado y concordante con lo que hoy en día se espera de un médico general, pero, además, egresaremos con la experiencia y quizás ventaja de haber vivido y aprendido en carne propia de esta crisis sanitaria.

A modo de cierre, compartimos un extracto de la reflexión sobre la experiencia vivida durante el voluntariado de uno de los autores, mostrando el aspecto más humano de ésta. "La pandemia por COVID-19 nos ha enseñado que tenemos una fuerza y resiliencia inagotable como seres humanos, lo que ha generado equipos de salud mucho más unidos y transversales. Aprendimos a sufrir, sí, a sufrir y a no tener miedo de expresar nuestro sentir. Si eres personal de salud y estás leyendo esto, también seguramente te acordaste, como me pasó a mí, por qué amabas la Medicina. Cuando ese paciente se recuperó y le fuiste a dar el alta, te tomó la mano y te dio las gracias. Eso es todo para mí, es tan gratificante que hace que todo el trabajo y sufrimiento valga la pena. Se me olvida todo lo malo. $Y$ me siento orgullosa y agradecida, de todos los profesionales de la salud, especialmente de mis compañeros internos que seguimos trabajando para combatir esta enfermedad todos los días. Porque ese es el espíritu que necesitamos para ser los médicos que nuestro futuro necesita, y es muy esperanzador verlo todos los días en los pasillos del hospital".

\section{Fuentes de financiamiento}

Declaramos no haber recibido financiamiento externo.

\section{Contribuciones y conflictos de interés declarados por los autores}

El autor Max Andresen es editor en jefe de esta revista. Declaramos esto como un conflicto de interés.

\section{Referencias}

Chandratre, S. (2020). Medical Students and COVID-19: Challenges and Supportive Strategies. Journal Of Medical Education And Curricular Development, 7.

Flotte, T., Larkin, A., Fischer, M., Chimienti, S., DeMarco, D., Fan, P., \& Collins, M. (2020). Accelerated Graduation and the Deployment of New Physicians During the COVID-19 Pandemic. Academic Medicine, Publish Ahead of Print.

Gill D, Whitehead C. \& Wondimagegn D. (2020). Challenges to medical education at a time of physical distancing. The Lancet, 396, 77-79.

Kinder F, \& Harvey A. (2020). Covid-19: the medical students responding to the pandemic. BMJ, 2160.

Miller D, Pierson L. \& Doernberg, S. (2020). The Role of Medical Students During the COVID-19 Pandemic. Annals Of Internal Medicine 173, 145-146.

Moszkowicz D, Duboc H, Dubertret C, Roux D \& Bretagnol F. (2020) Daily medical education for confined students during COVID-19 pandemic: A simple videoconference solution. Clin Anat. 33, 927-928.

Rose S. (2020). Medical Student Education in the Time of COVID-19. JAMA 323, 2131-2132.

Roskvist R, Eggleton K. \& Goodyear-Smith F. (2020). Provision of e-learning programmes to replace undergraduate medical students clinical general practice attachments during COVID-19 stand-down. Education For Primary Care, 31, 247-254

Santos J, Chang D, Robbins K, Cam E, Garbuzov A. \& Miyakawa-Liu, M. et al. (2020). Answering the Call: Medical Students Reinforce Health System Frontlines Through Ochsner COVID-19 Hotline. Ochsner Journal 20, 144-145. 